\title{
Treatment method and prognostic factors of chondrosarcoma: based on Surveillance, Epidemiology, and End Results (SEER) database
}

\author{
Kun-Chi Hua, Yong-Cheng Hu \\ Department of Orthopedic Oncology, Tianjin Hospital, Tianjin, China \\ Contributions: (I) Conception and design: KC Hua; (II) Administrative support: YC Hu; (III) Provision of study materials or patients: KC Hua; (IV) \\ Collection and assembly of data: KC Hua; (V) Data analysis and interpretation: KC Hua; (VI) Manuscript writing: All authors; (VII) Final approval \\ of manuscript: All authors. \\ Correspondence to: Yong-Cheng Hu. Department of Orthopedic Oncology, Tianjin Hospital, Tianjin 300211, China. Email: HuYC62@163.com.
}

Background: Chondrosarcoma is a malignant tumor originating from cartilage tissue. It is the second most malignant bone tumor, accounting for about $10 \%$ to $15 \%$ of all primary bone tumors. So far, there have been no reports of large-scale clinical statistics on the relationship between non-surgical treatment and prognosis in patients with chondrosarcoma.

Methods: Through the search of the Surveillance, Epidemiology, and End Results (SEER) database, chondrosarcoma patients registered between January 1, 2004 and December 31, 2016 were selected as research goals. Univariate analysis of overall survival (OS) and chondrosarcoma-specific survival (CSSS) by Kaplan-Meier survival analysis and log-rank test. Mapping Kaplan-Meier curves for prognostic factors that are significant for OS and CSSS in patients with chondrosarcoma. After univariate analysis, the prognostic factors that have a significant effect on the prognosis were included in the multivariate Cox regression analysis, and the independent factors that affected the prognosis were screened.

Results: A total of 1,128 patients with chondrosarcoma were included in the study. Univariate analysis showed that prognostic factors such as age, gender, primary site, histological type, grade, tumor size, metastasis, surgery, radiotherapy, chemotherapy and treatment method had significant effects on all-cause mortality (ACM) and chondrosarcoma-specific mortality (CSSM). These factors were included in the multivariate Cox regression analysis. The results showed age, primary site, histological type, grade, tumor size, metastasis, and treatment method were independent factors affecting ACM and CSSM.

Conclusions: This study found that although non-surgical treatment of chondrosarcoma has made great progress, at present, it is still not considered that non-surgical treatment can significantly improve the prognosis. There are many factors affecting the prognosis of chondrosarcoma, including age, primary site, histological type, grade, tumor size, distant metastasis and treatment method. In the future, more samples and more detailed data will be needed to study the treatment of chondrosarcoma and to analyze the important factors affecting prognosis through big data analysis.

Keywords: Chondrosarcoma; Surveillance, Epidemiology, and End Results (SEER); treatment; prognostic factor

Submitted Jan 09, 2020. Accepted for publication Jun 05, 2020.

doi: $10.21037 /$ tcr-20-357

View this article at: http://dx.doi.org/10.21037/tcr-20-357 


\section{Introduction}

Chondrosarcoma is a malignant tumor originating from cartilage tissue $(1,2)$. It is the second most malignant bone tumor, accounting for about $10 \%$ to $15 \%$ of all primary bone tumors (3-5). The annual incidence of about 200,000 people, accounting for about $20 \%$ of bone malignant tumor in the United States (US) (6-9). It can occur at any age, with an average age of onset of 50 years, and more men than women $(55 \%: 45 \%)(10,11)$. The chondrosarcoma of the axis is most common in the pelvis (12-14). The femur in the long bones of the extremities is most often involved $(15,16)$. Another $10 \%$ of chondrosarcoma occurs in soft tissues, mostly mucinous chondrosarcoma.

Chondrosarcoma is characterized by the production of hyaline cartilage by tumor cells, a tumor that is less malignant and slow to grow but is prone to recurrence in the absence of treatment (17). Chondrosarcoma has poor blood supply and lymphatic circulation, and is not sensitive to traditional chemotherapy (CT) drugs and radiotherapy (RT) (18). At present, extensive resection is still the main treatment, and the 10 -year survival rate is $30-80 \%$ (10). However, with the deepening of research on radiosensitizers and the development of genetic engineering in recent years, many scholars have gradually changed their understanding $(19,20)$. More and more studies have suggested that RT and neoadjuvant CT have certain clinical significance for the treatment of chondrosarcoma (19-21).

So far, there have been no reports of large-scale clinical statistics on the relationship between non-surgical treatment and prognosis in patients with chondrosarcoma. In addition, the incidence of primary bone tumors is significantly less than that of other systems. There are fewer clinical treatment centers specializing in bone tumor research in China, and there is a lack of large-scale clinical statistics. In order to solve the problem of insufficient clinical data, we collected information on patients with chondrosarcoma from the Surveillance, Epidemiology, and End Results (SEER) database managed by the National Cancer Institute. The database contains approximately $30 \%$ of the US population's cancer diagnosis, treatment and survival data, and global oncology researchers have obtained partial data by applying to provide a good source of data for this study $(22,23)$.

This article retrospectively analyzed the data of 1,128 patients with chondrosarcoma in the SEER database to study the patients undergoing surgery, RT and CT. In addition, 9 factors including age, gender, ethnicity, tumor location, tumor size, grade classification, histological type, distant metastasis, and treatment were analyzed to screen out factors affecting prognosis. Provide more direct evidence for the choice of diagnosis and treatment method to achieve the goal of improving the prognosis of patients with chondrosarcoma. We present the following article in accordance with the STROBE reporting checklist (available at http://dx.doi.org/10.21037/tcr-20-357).

\section{Methods}

\section{Data collection}

SEER ${ }^{*}$ Stat software, version 8.3.6 was used to access the SEER 18 Regs Custom Data (with additional treatment field), Nov 2018 Sub (1973-2016 varying) database using SEER*Stat's client-server mode. A total of 1,128 patients with chondrosarcoma in the US diagnosed between January 1, 2004 and December 31, 2016. Patients confirmed before 2004 were excluded because demographic and treatment information was incomplete. Patients were excluded if they had unknown survival time, were unknown vital status, were diagnosed at autopsy, or had unknown treatment method.

\section{Inclusion codes and criteria}

The primary endpoints of the study were overall survival (OS) and chondrosarcoma-specific survival (CSSS). In this study, we classified patients according to the following factors, such as age at diagnosis ( $\leq 55,56-73, \geq 74$ years), sex (female, male), race (White, Black, others), primary site (upper limb, lower limb, axial, other), histological type (conventional chondrosarcoma, myxoid chondrosarcoma, dedifferentiated chondrosarcoma, other), grade (I, II, III, IV), tumor size $(\leq 72,73-117, \geq 118 \mathrm{~mm})$, metastasis (yes, no), surgery (yes, no), RT (yes, no), CT (yes, no), and treatment method (surgery, no surgery and no RT and no CT, RT, CT, surgery and RT, surgery and CT, RT and CT, surgery and RT and CT).

\section{Statistical analysis}

The clinicopathological data of patients who received/not received surgery, received/not received RT, and received/ not received CT were compared by chi-square test. In addition, the chi-square test was used to compare OS and CSSS for each prognostic factor of chondrosarcoma patients. To better set the cut-off value of the continuity 
variables (age and tumor size), use $\mathrm{X}$-tile software for analysis. Mapping Kaplan-Meier curves for prognostic factors that are significant differences for OS and CSSS in patients with chondrosarcoma. After univariate analysis, the prognostic factors that have a significant effect on the prognosis were included in the multivariate Cox regression analysis, and the independent factors that affected the prognosis were screened. The $\mathrm{P}$ value $<0.05$ was considered to be significant. All statistical analysis is completed by Statistical package for the social sciences (SPSS, version 23.0; IBM Corp., Armonk, NY, USA).

\section{Results}

\section{Determination cut-off value of age and tumor size}

X-tile software analysis results (Figure 1) show that the best cut-off values for age in patients with chondrosarcoma are 55 and 74 years, thus dividing the patient's age into three groups ( $\leq 55,56-73, \geq 74$ years). The optimal cut-off values for tumor size were 72 and $118 \mathrm{~mm}$, thus dividing the tumor size into three groups $(\leq 72,73-117, \geq 118 \mathrm{~mm})$.

\section{Clinicopathological features of patients with chondrosarcoma}

A total of 1,128 patients with chondrosarcoma were included in the study (Figure 2), aged 2 to 98 years, with an overall median survival time of 35 months. Among them, the majority of patients aged $\leq 55$ years $(568,50.4 \%)$ and the median survival time was the longest (38 months). Four hundred and ninety-three women $(43.7 \%)$, median survival time was 36 months, 635 patients were male patients (56.3\%), median survival time was 34 months, male to female ratio was 1.29:1. White account for the vast majority (989, 87.7\%), and black have the longest median survival time (35.5 months). The highest incidence was in the axial bone $(468,41.5 \%)$, while the median survival time of the upper limbs was the longest (36.5 months). Conventional chondrosarcoma is dominant $(934,82.8 \%$ ) with the longest median survival time (38 months). The incidence of grade I and grade II was close (35.4\% vs. $42.6 \%$ ), the incidence of grade III and grade IV was close (12.9\% vs. $9.1 \%)$, and the median survival time of grade I was the longest (42.0 months). Tumor size $\leq 72 \mathrm{~mm}$ is the most (554, 49.1\%) and the median survival time is the longest (39.5 months). Patients with no distant metastases were the most $(1,060$, $94.0 \%$ ) with the longest median survival time (36.0 months).
The median survival time of patients undergoing surgery was longer than that of patients who did not undergo surgery (37.0 vs. 18.0 months). The median survival time of patients who did not receive RT was longer than those who received RT (36.0 vs. 28.0 months). The median survival time of patients who did not receive CT was longer than that of patients who received CT (36.0 vs. 19.0 months). Among the treatment method options, patients with only surgery had the highest median survival time (39.0 months) (Table 1).

\section{Selection of surgery, RT and CT in patients with chondrosarcoma}

There were significant differences in factors such as age, race, primary site, grade, tumor size, and distant metastasis in the choice of surgery. The highest proportion of white patients undergoing surgery $(90.9 \%)$. Chondrosarcoma appears in patients with axial bone, and the proportion of patients undergoing surgery is slightly lower than that of other patients $(86.8 \%)$. From grade I to IV, the proportion of patients undergoing surgery gradually decreased. Similarly, as the tumor size increased, the proportion of patients undergoing surgery gradually decreased. The proportion of patients who did not have distant metastases was significantly higher than those with distant metastases (92.3\% vs. $52.9 \%)$.

In the choice of RT, there are significant differences in factors such as primary site, histological type, grade, and distant metastasis. The proportion of patients with chondrosarcoma in the upper and lower extremities receiving $\mathrm{RT}$ was close $(6.0 \%$ vs. $5.8 \%)$ and lower than that of the central axis (16.7\%). Myxoid chondrosarcoma patients had the highest proportion of RT (25.9\%). From grade I to IV, the proportion of patients receiving RT increased gradually. The proportion of patients with distant metastases receiving RT was significantly higher than those without distant metastasis (25.0\% vs. 13.4\%).

In the choice of CT, there are significant differences in factors such as histological type, grade, tumor size, and distant metastasis. Among them, dedifferentiated chondrosarcoma patients had the highest proportion of CT (33.0\%). Grade IV patients had the highest proportion of CT (35.9\%). Patients with tumor size $\geq 118 \mathrm{~mm}$ had the highest proportion of CT (13.2\%). The proportion of patients with distant metastases receiving CT was significantly higher than that of patients without distant metastases $(41.2 \%$ vs. $5.3 \%$ ) (Table 2$)$. 


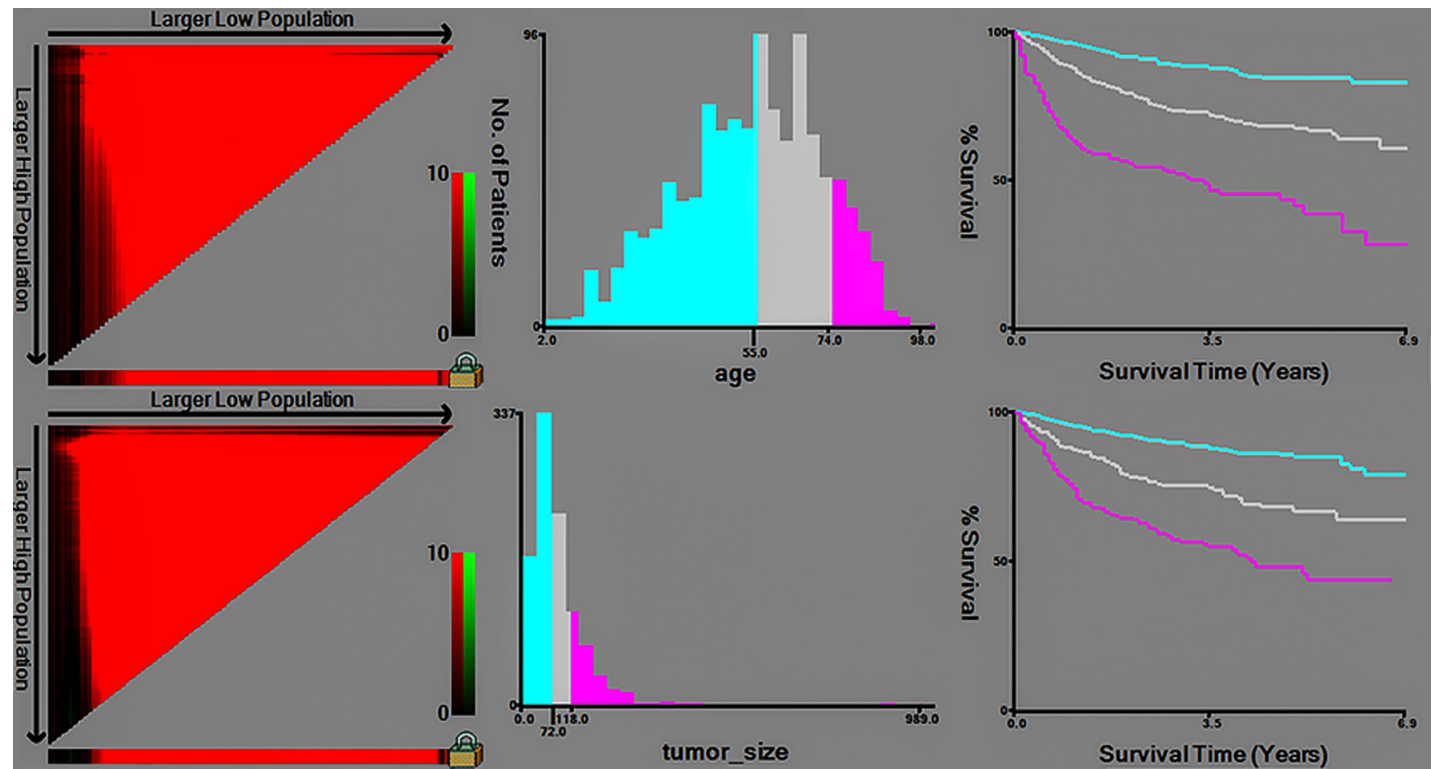

Figure $1 \mathrm{X}$-tile software analysis SEER database chondrosarcoma patients the best age and tumor size cutoff value. SEER, Surveillance, Epidemiology, and End Results.

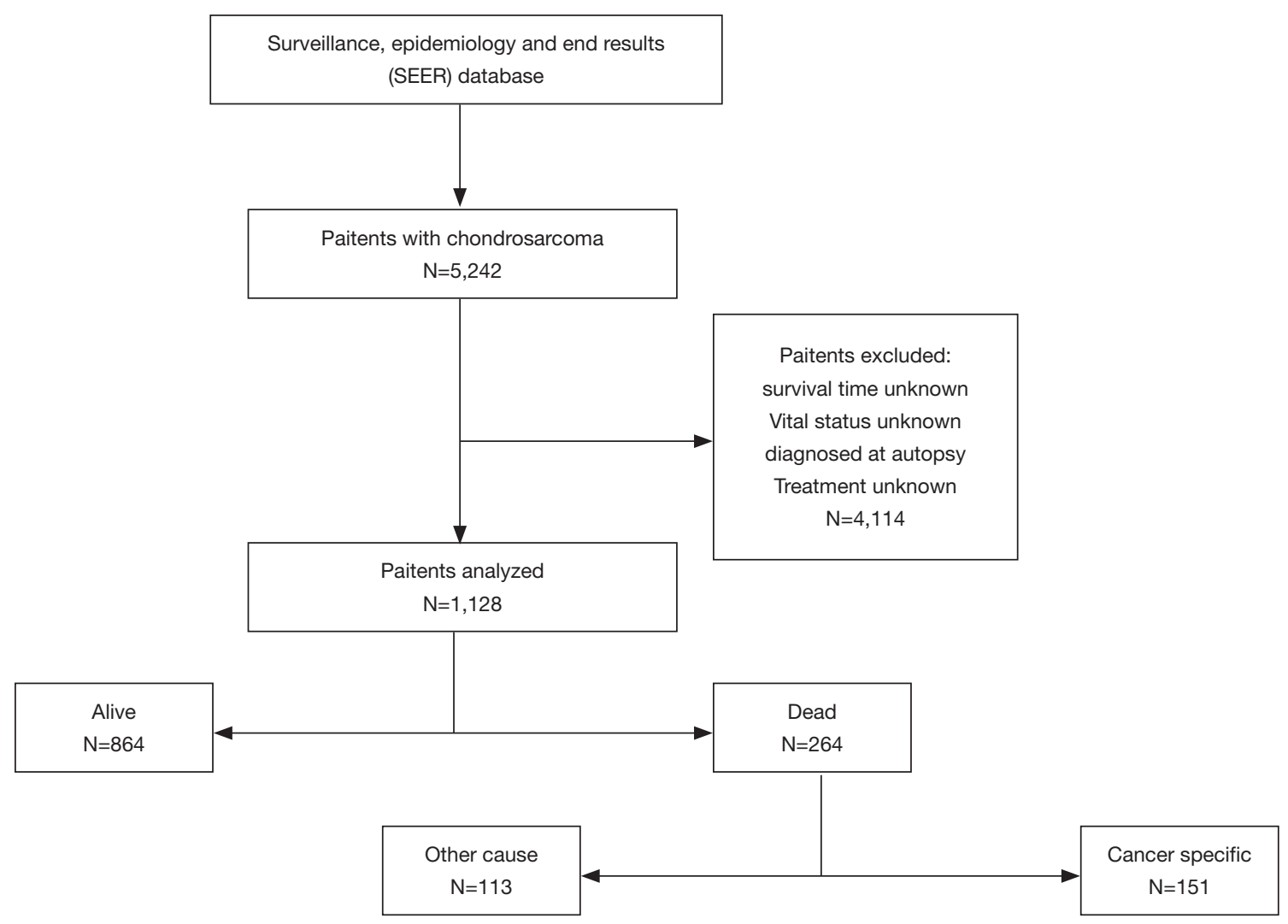

Figure 2 Flowchart of patient identification and selection. 
Table 1 Survival time for each prognostic factor and categorical variable

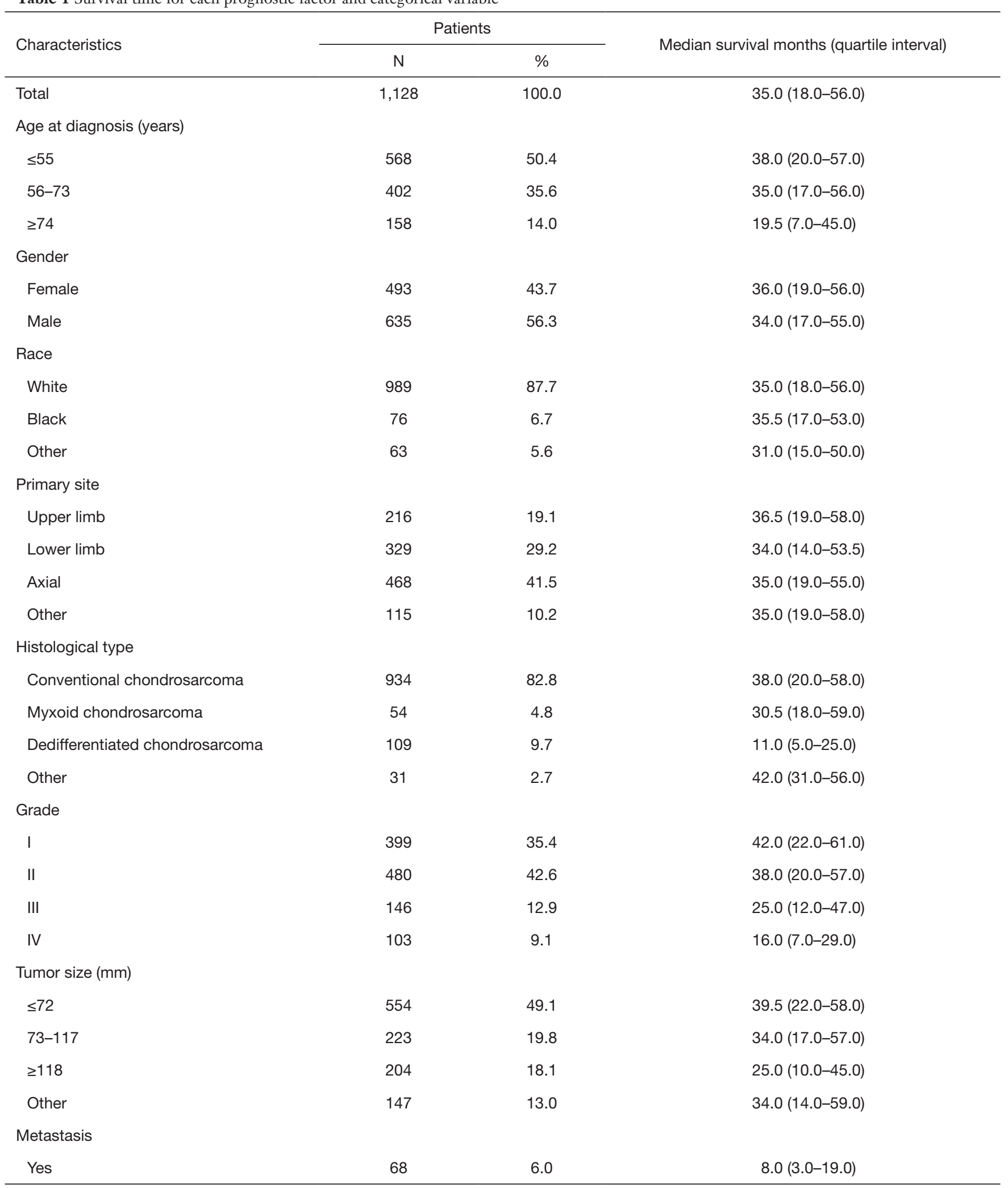

Table 1 (continued) 
Table 1 (continued)

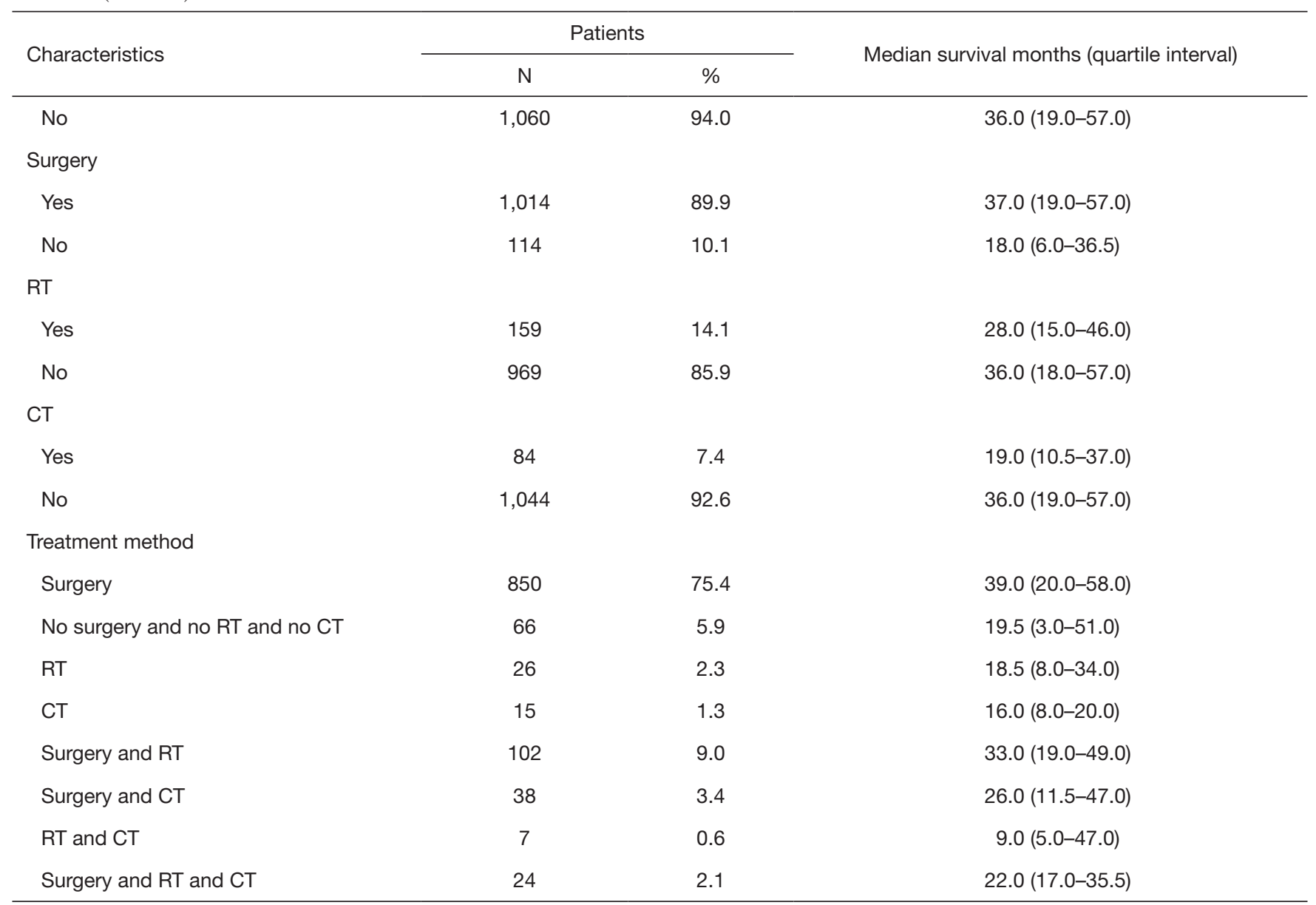

$\mathrm{RT}$, radiotherapy; CT, chemotherapy.

\section{Prognostic factors and categorical variables of all-cause mortality (ACM) and chondrosarcoma-specific mortality (CSSM)}

In addition to ethnicity, there were significant differences in prognostic factors and categorical variables between ACM and CSSM. Patients with age $\geq 74$ had the highest ACM and CSSM (52.5\% and 29.9\%, respectively). In ACM and CSSM, male is higher than female $(26.8 \%$ vs. $19.1 \%$, $17.4 \%$ vs. $11.4 \%$, respectively). In ACM and CSSM, lower limb was the highest $(29.5 \%$ and $21.1 \%$, respectively). Dedifferentiated chondrosarcoma patients with the highest ACM and CSSM (76.1\% and 69.0\%, respectively). Grade IV had the highest ACM and CSSM (69.9\% and 61.7\%, respectively). Patients with tumor size $\geq 118 \mathrm{~mm}$ had the highest ACM and CSSM (44.1\% and 33.3\%, respectively).
Patients with distant metastases had higher ACM and CSSM than those without metastasis $(83.8 \%$ vs. $19.5 \%$, $81.7 \%$ vs. $10.7 \%$, respectively). Patients who did not undergo surgery had higher ACM and CSSM than those who underwent surgery (54.4\% vs. $19.9 \%, 43.5 \%$ vs. $12.0 \%$, respectively). In ACM and CSSM, patients receiving RT were higher than those who did not receive RT $(39.0 \%$ vs. $20.8 \%, 28.1 \%$ vs. $12.8 \%$, respectively). Patients receiving CT had higher ACM and CSSM than those without CT (64.3\% vs. $20.1 \%, 60.5 \%$ vs. $11.2 \%$, respectively). In the choice of treatment options, Patients only receiving CT have the highest ACM and CSSM (86.7\% and $85.7 \%$, respectively), whereas patients with surgery only had the lowest ACM and CSSM (16.4\% and 8.8\%, respectively) (Table 3). 


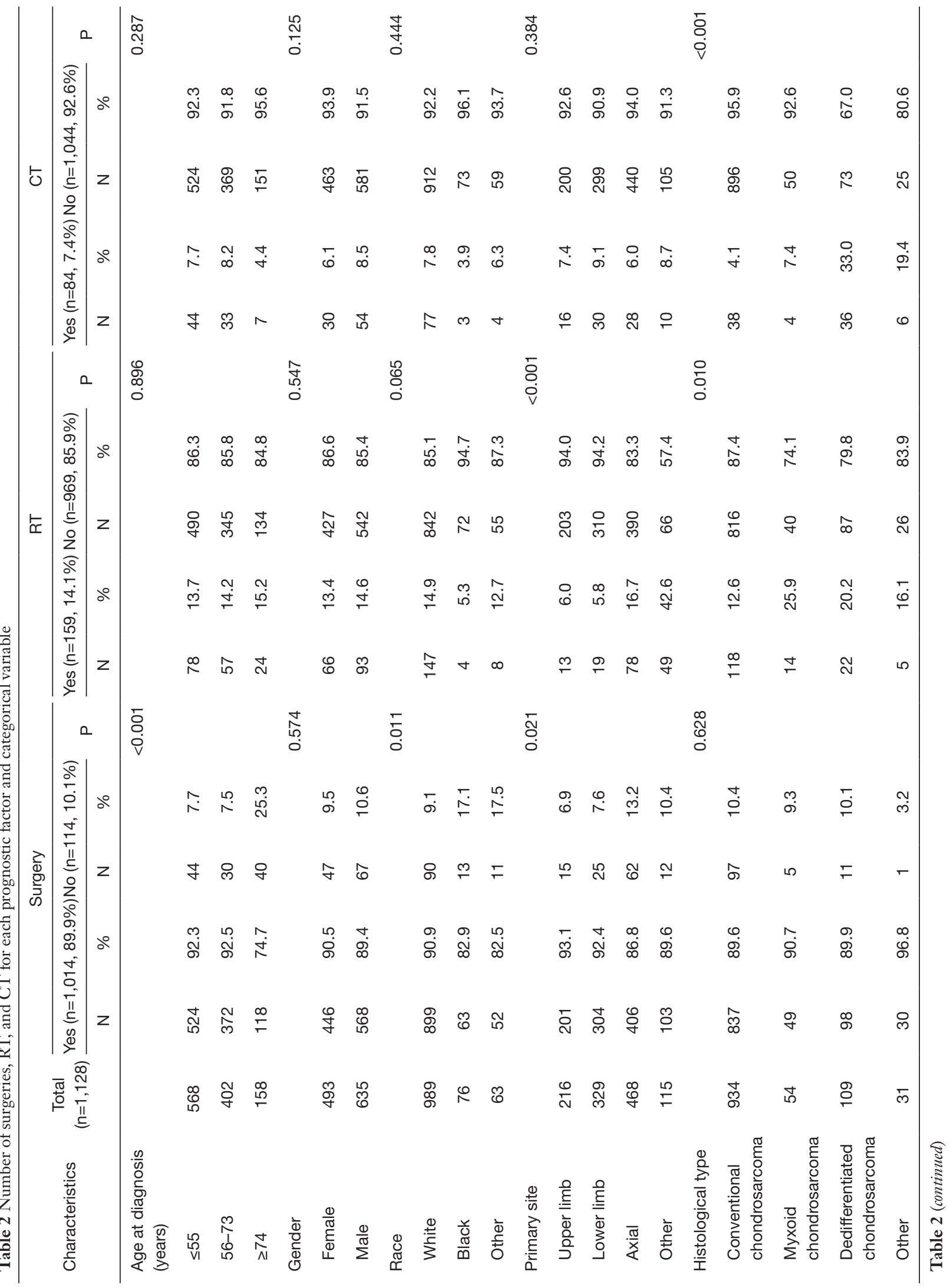




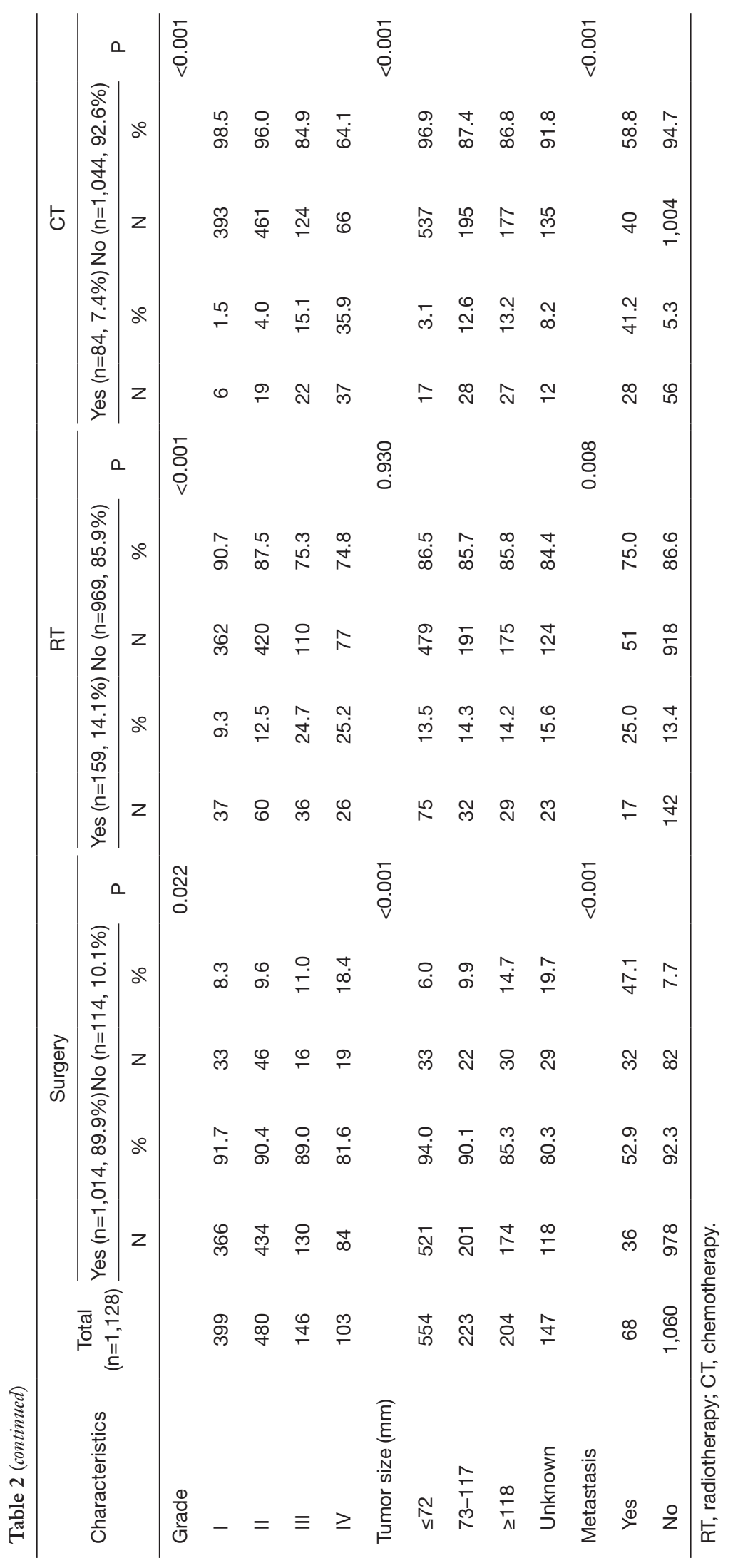




\section{Univariate analysis}

Age, gender, primary site, histological type, grade, tumor size, metastasis, surgery, RT, CT, and treatment method were significantly different, indicating that these prognostic factors were significant associated with ACM and CSSM (Table 4). In addition, we plotted the Kaplan-Meier survival curves for each prognostic factor associated with ACM and $\operatorname{CSSM}$ (Figure 3).

\section{Multivariate analysis}

The Kaplan-Meier survival analysis was used to analyze the independent factors that have significant correlation between ACM and CSSM. The results showed age, primary site, histological type, grade, tumor size, metastasis, and treatment method were independent factors affecting ACM and CSSM (Table 4).

\section{Discussion}

Chondrosarcoma is not a common disease, so there is no extensive and in-depth study of its diagnosis and treatment. It has been previously thought that chondrosarcoma is not sensitive to all CT and RT, and patients need to undergo surgical resection of the primary site of extensive lesions. Patients with primary lesion resection have inevitable disability and dysfunction, and surgical resection does not make much sense for tumors that have metastases distantly (24).

\section{Treatment method}

At present, in the surgical treatment of chondrosarcoma, limb salvage surgery has become the first choice for orthopedic surgeons, and adjuvant therapy has become an indispensable means of treatment for chondrosarcoma, but no clear conclusions have been drawn on the efficacy of adjuvant therapy. In this study, the longest survival time was still only surgery (39 months). However, surgery with RT (33 months) or surgery with CT (26 months) did not prolong survival time. In addition, both ACM and CSSM, patients with only surgery were the lowest $(16.4 \%$ and $8.8 \%$, respectively). In the multivariate analysis, surgery and RT or surgery and CT have an increased risk of death compared to only surgery. In addition, in CSSM, the hazard ratios (HR) for surgery and CT reached 4.510, which is almost twice the HR for surgery and RT. There are two reasons for this.
On the one hand, patients with high degree of malignancy generally choose the combination of surgery and adjuvant therapy, which is also reflected in our study, from grade I to IV, the proportion of patients receiving RT or CT is gradually increasing, and the risk of death in patients with highly malignant tumors is high. Therefore, the effect of adjuvant therapy cannot be clearly demonstrated. On the other hand, chondrosarcoma is not sensitive to RT and CT and has been supported by many studies. Although studies have been conducted on deeper studies of radiosensitizers or CT regimens for chondrosarcoma, so far, no satisfactory drugs have emerged. Therefore, this study does not support the idea that adjuvant therapy can significantly improve the prognosis of patients with chondrosarcoma.

\section{Prognostic factor}

In this study, age was significantly correlated with prognosis, with older patients having worse prognosis. Giuffrida et al. (9) analyzed data from 2,890 patients with chondrosarcoma in the SEER database. The prognosis of patients older than 50 years was relatively poor, the 30 -year survival rate was only $20 \%$. While the age less than 50 years old, the 30 year survival rate was greater than $60 \%$. The prognosis of female chondrosarcoma is better than that of males. The female 30 -year survival rate is $78.6 \%$, while the male 30 year survival rate is $68.7 \%$, which is statistically different.

The primary site of the tumor showed significant correlation with the prognosis. The HR of lower limb or axial bone was higher than that of upper limb, and the prognosis was poor. At present, there is no comprehensive study on the prognosis of chondrosarcoma of the upper and lower limbs. After analyzing 227 cases of chondrosarcoma, Lee et al. (8) considered that the prognosis of chondrosarcoma occurring in the limbs is better than other sites, with higher survival rate and lower recurrence rate. Fiorenza et al. (25) analyzed 153 cases of non-metastatic chondrosarcoma and found that the 5- and 10-year survival rates of osteochondromatosis of the extremities were $81 \%$ and $71 \%$, respectively, and the 5 - and 10 -year survival rates of pelvic chondrosarcoma were $69 \%$ and $59 \%$, respectively. Although the survival rate of patients with chondrosarcoma of the extremities is higher, it is not statistically significant.

Histological type was significantly associated with prognosis, with dedifferentiated chondrosarcoma having the worst prognosis. Conventional chondrosarcoma accounts for $85 \%$ of all chondrosarcoma, including primary chondrosarcoma and secondary chondrosarcoma. Myxoid 
Table 3 All-cause mortality and chondrosarcoma-specific mortality for each prognostic factor and categorical variable

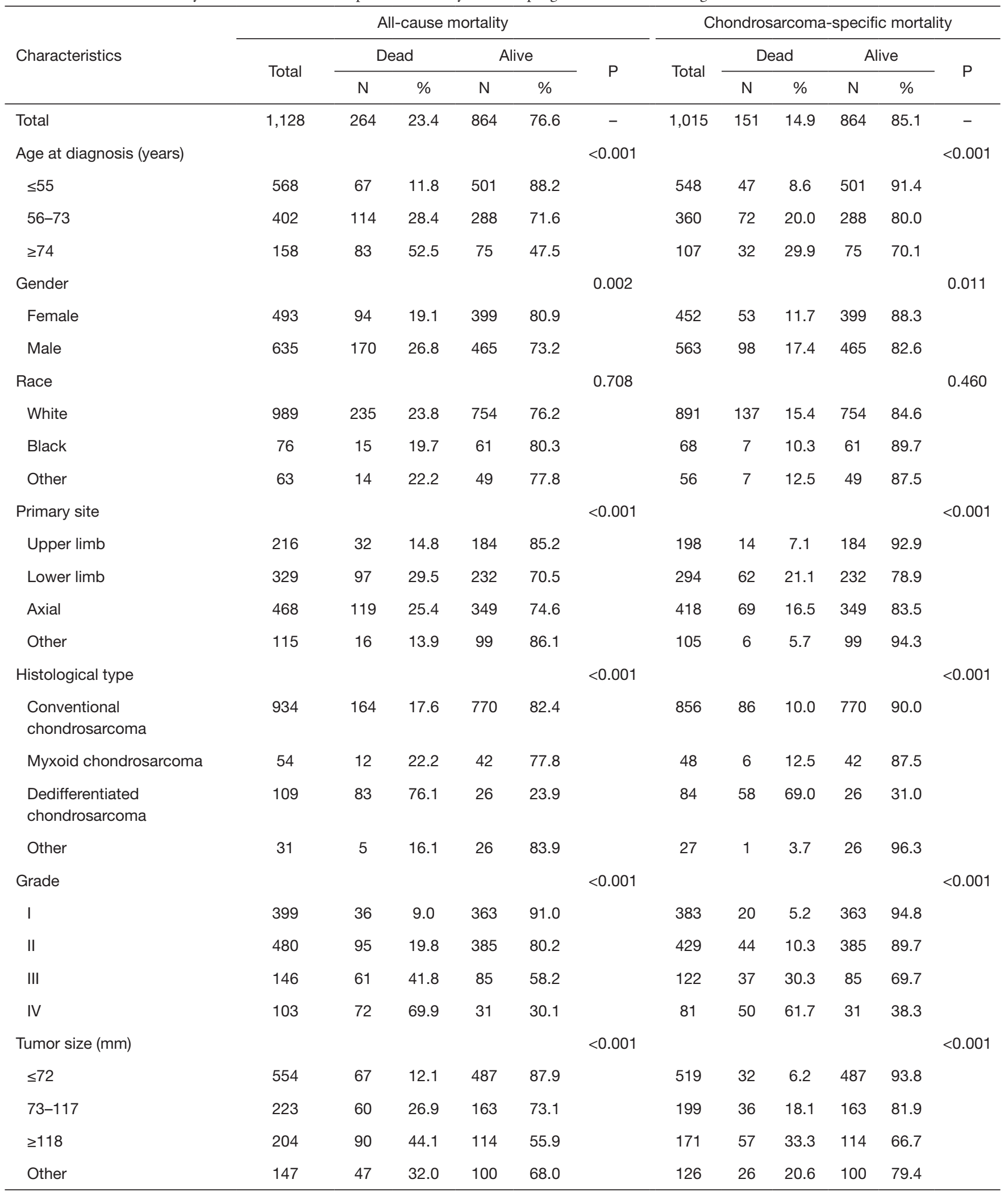

Table 3 (continued) 
Table 3 (continued)

\begin{tabular}{|c|c|c|c|c|c|c|c|c|c|c|c|c|}
\hline \multirow{2}{*}{ Characteristics } & \multicolumn{6}{|c|}{ All-cause mortality } & \multicolumn{6}{|c|}{ Chondrosarcoma-specific mortality } \\
\hline & Total & $\mathrm{N}$ & $\%$ & $\mathrm{~N}$ & $\%$ & $\mathrm{P}$ & Total & $\mathrm{N}$ & $\%$ & $\mathrm{~N}$ & $\%$ & $\mathrm{P}$ \\
\hline Metastasis & & & & & & $<0.001$ & & & & & & $<0.001$ \\
\hline Yes & 68 & 57 & 83.8 & 11 & 16.2 & & 60 & 49 & 81.7 & 11 & 18.3 & \\
\hline Surgery & & & & & & $<0.001$ & & & & & & $<0.001$ \\
\hline Yes & 1014 & 202 & 19.9 & 812 & 80.1 & & 923 & 111 & 12.0 & 812 & 88.0 & \\
\hline No & 114 & 62 & 54.4 & 52 & 45.6 & & 92 & 40 & 43.5 & 52 & 56.5 & \\
\hline RT & & & & & & $<0.001$ & & & & & & $<0.001$ \\
\hline CT & & & & & & $<0.001$ & & & & & & $<0.001$ \\
\hline Yes & 84 & 54 & 64.3 & 30 & 35.7 & & 76 & 46 & 60.5 & 30 & 39.5 & \\
\hline No & 1044 & 210 & 20.1 & 834 & 79.9 & & 939 & 105 & 11.2 & 834 & 88.8 & \\
\hline Treatment method & & & & & & $<0.001$ & & & & & & $<0.001$ \\
\hline Surgery & 850 & 139 & 16.4 & 711 & 83.6 & & 780 & 69 & 8.8 & 711 & 91.2 & \\
\hline $\begin{array}{l}\text { No surgery and no RT and no } \\
\text { CT }\end{array}$ & 66 & 27 & 40.9 & 39 & 59.1 & & 52 & 13 & 25.0 & 39 & 75.0 & \\
\hline RT & 26 & 17 & 65.4 & 9 & 34.6 & & 19 & 10 & 52.6 & 9 & 47.4 & \\
\hline
\end{tabular}

$\mathrm{RT}$, radiotherapy; CT, chemotherapy.

chondrosarcoma is a subtype of primary chondrosarcoma isolated from conventional chondrosarcoma. From the cytogenetic analysis, it is caused by the translocation of the homologous chromosome 9 and the 22 position (26). Dedifferentiated chondrosarcoma is one of the highly malignant subtypes of chondrosarcoma. The tumor is highly prone to metastasis, especially lung metastasis. The mortality rate is high and the prognosis is poor. If metastasis has occurred at the time of diagnosis and the tumor size $>8 \mathrm{~cm}$, the prognosis is even less optimistic (27).

Grade and tumor size showed significant correlation with prognosis. Patients with poor differentiation or large tumor size have a poor prognosis. The higher the grade, the lower the degree of tumor tissue differentiation, which means that the degree of malignancy of the tumor increases. The expansion of the tumor size means that the tumor tissue is more difficult to remove completely, the possibility of residual and local recurrence increases, and the risk of distant metastasis is greatly improved. This suggests that the surgeon should strictly follow the guiding principle of "complete resection" when faced with poorly differentiated and large chondrosarcoma.

Distant metastasis showed significant correlation with prognosis, and metastasis meant poor prognosis. It is 
Table 4 Results of univariate and multivariate analysis of each prognostic factor and categorical variable

\begin{tabular}{|c|c|c|c|c|c|c|c|c|}
\hline Characteristics & \multicolumn{3}{|c|}{ All-cause mortality } & $\begin{array}{l}\text { log-rank } \\
\mathrm{P} \text { value }\end{array}$ & \multicolumn{3}{|c|}{ Chondrosarcoma-specific mortality } & $\begin{array}{c}\text { log-rank } \\
\mathrm{P} \text { value }\end{array}$ \\
\hline Age at diagnosis (years) & & & & $<0.001$ & & & & $<0.001$ \\
\hline$\leq 55$ & 1.000 (reference) & & & & 1.000 (reference) & & & \\
\hline $56-73$ & 2.041 & $1.4793-2.8171$ & $<0.001$ & & 1.769 & $1.1788-2.6544$ & 0.006 & \\
\hline Gender & & & & 0.005 & & & & 0.016 \\
\hline Female & 1.000 (reference) & & & & 1.000 (reference) & & & \\
\hline Male & 1.289 & $0.9967-1.6671$ & 0.053 & & 1.279 & $0.9006-1.8156$ & 0.169 & \\
\hline Race & & & & 0.531 & & & & 0.275 \\
\hline Other & NA & NA & NA & & NA & NA & NA & \\
\hline Primary site & & & & $<0.001$ & & & & $<0.001$ \\
\hline Upper limb & 1.000 (reference) & & & & 1.000 (reference) & & & \\
\hline Lower limb & 1.345 & $0.8875-2.0377$ & 0.162 & & 1.979 & $1.0784-3.6308$ & 0.028 & \\
\hline Axial & 1.445 & $0.9549-2.1859$ & 0.082 & & 2.073 & $1.1278-3.8085$ & 0.019 & \\
\hline Other & 0.903 & $0.4775-1.7071$ & 0.753 & & 0.692 & $0.2532-1.8895$ & 0.472 & \\
\hline Histological type & & & & $<0.001$ & & & & $<0.001$ \\
\hline $\begin{array}{l}\text { Conventional } \\
\text { chondrosarcoma }\end{array}$ & 1.000 (reference) & & & & 1.000 (reference) & & & \\
\hline Grade & & & & $<0.001$ & & & & $<0.001$ \\
\hline I & 1.000 (reference) & & & & 1.000 (reference) & & & \\
\hline II & 1.685 & $1.1346-2.5025$ & 0.009 & & 1.348 & $0.7793-2.3300$ & 0.286 & \\
\hline III & 2.503 & $1.5603-4.0147$ & $<0.001$ & & 2.151 & $1.1350-4.0756$ & 0.019 & \\
\hline IV & 4.054 & $2.4294-6.7634$ & $<0.001$ & & 4.434 & $2.2438-8.7627$ & $<0.001$ & \\
\hline Tumor size $(\mathrm{mm})$ & & & & $<0.001$ & & & & $<0.001$ \\
\hline$\leq 72$ & 1.000 (reference) & & & & 1.000 (reference) & & & \\
\hline $73-117$ & 1.370 & $0.9433-1.9886$ & 0.098 & & 1.343 & $0.8031-2.2446$ & 0.261 & \\
\hline$\geq 118$ & 2.505 & $1.7709-3.5430$ & $<0.001$ & & 2.924 & $1.8080-4.7281$ & $<0.001$ & \\
\hline Other & 2.034 & $1.3691-3.0229$ & $<0.001$ & & 2.363 & $1.3639-4.0940$ & 0.002 & \\
\hline
\end{tabular}

Table 4 (continued) 
Table 4 (continued)

\begin{tabular}{|c|c|c|c|c|c|c|c|c|}
\hline \multirow{2}{*}{ Characteristics } & \multicolumn{3}{|c|}{ All-cause mortality } & \multirow{2}{*}{$\begin{array}{l}\text { log-rank } \\
\mathrm{P} \text { value }\end{array}$} & \multicolumn{3}{|c|}{ Chondrosarcoma-specific mortality } & \multirow{2}{*}{$\begin{array}{l}\text { log-rank } \\
\mathrm{P} \text { value }\end{array}$} \\
\hline & HR & $95 \% \mathrm{Cl}$ & $P$ value & & $\mathrm{HR}$ & $95 \% \mathrm{Cl}$ & $P$ value & \\
\hline Metastasis & & & & $<0.001$ & & & & $<0.001$ \\
\hline Yes & 1.000 (reference) & & & & 1.000 (reference) & & & \\
\hline No & 0.335 & $0.2323-0.4825$ & $<0.001$ & & 0.232 & $0.1472-0.3645$ & $<0.001$ & \\
\hline Yes & 1.000 (reference) & & & & 1.000 (reference) & & & \\
\hline No & 1.114 & $0.3714-3.3392$ & 0.848 & & 1.028 & $0.3158-3.3479$ & 0.963 & \\
\hline RT & & & & $<0.001$ & & & & $<0.001$ \\
\hline Yes & 1.000 (reference) & & & & 1.000 (reference) & & & \\
\hline Yes & 1.000 (reference) & & & & 1.000 (reference) & & & \\
\hline No & 1.439 & $0.4128-5.0144$ & 0.568 & & 1.160 & $0.2864-4.6978$ & 0.835 & \\
\hline Treatment method & & & & $<0.001$ & & & & $<0.001$ \\
\hline Surgery & 1.000 (reference) & & & & 1.000 (reference) & & & \\
\hline $\begin{array}{l}\text { No surgery and no RT and } \\
\text { no CT }\end{array}$ & 2.102 & $0.6523-6.7739$ & 0.213 & & 2.092 & $0.5486-7.9804$ & 0.280 & \\
\hline RT & 3.035 & $0.9705-9.4931$ & 0.056 & & 4.244 & $1.2166-14.8015$ & 0.023 & \\
\hline CT & 1.875 & $0.5020-7.0019$ & 0.350 & & 1.801 & $0.4298-7.5454$ & 0.421 & \\
\hline
\end{tabular}

$\mathrm{RT}$, radiotherapy; CT, chemotherapy; $\mathrm{HR}$, hazard ratios; $\mathrm{Cl}$, confidence intervals; NA, not applicable.

worth noting that chondrosarcoma grows slowly, and its recurrence or metastasis can be 10 years after the first treatment, which means that the follow-up time for patients with chondrosarcoma is longer than that of general tumors, and monitoring the lesion should be more cautious.

\section{Limitations}

This study is based on a retrospective study conducted by the SEER database. Due to the limitations of the data included in the database itself, more detailed patient information is not available. We are unable to obtain a patient's specific surgical plan, detailed CT drugs, complications and tumor recurrence. In addition, there is no information on targeted therapy in the SEER database, which limits our ability to find more effective adjuvant treatments.

\section{Conclusions}

This study found that although non-surgical treatment of chondrosarcoma has made great progress, at present, it is still not considered that non-surgical treatment can significantly improve the prognosis. There are many factors affecting the prognosis of chondrosarcoma, including age, primary site, histological type, grade, tumor size, distant metastasis and treatment method. However, 

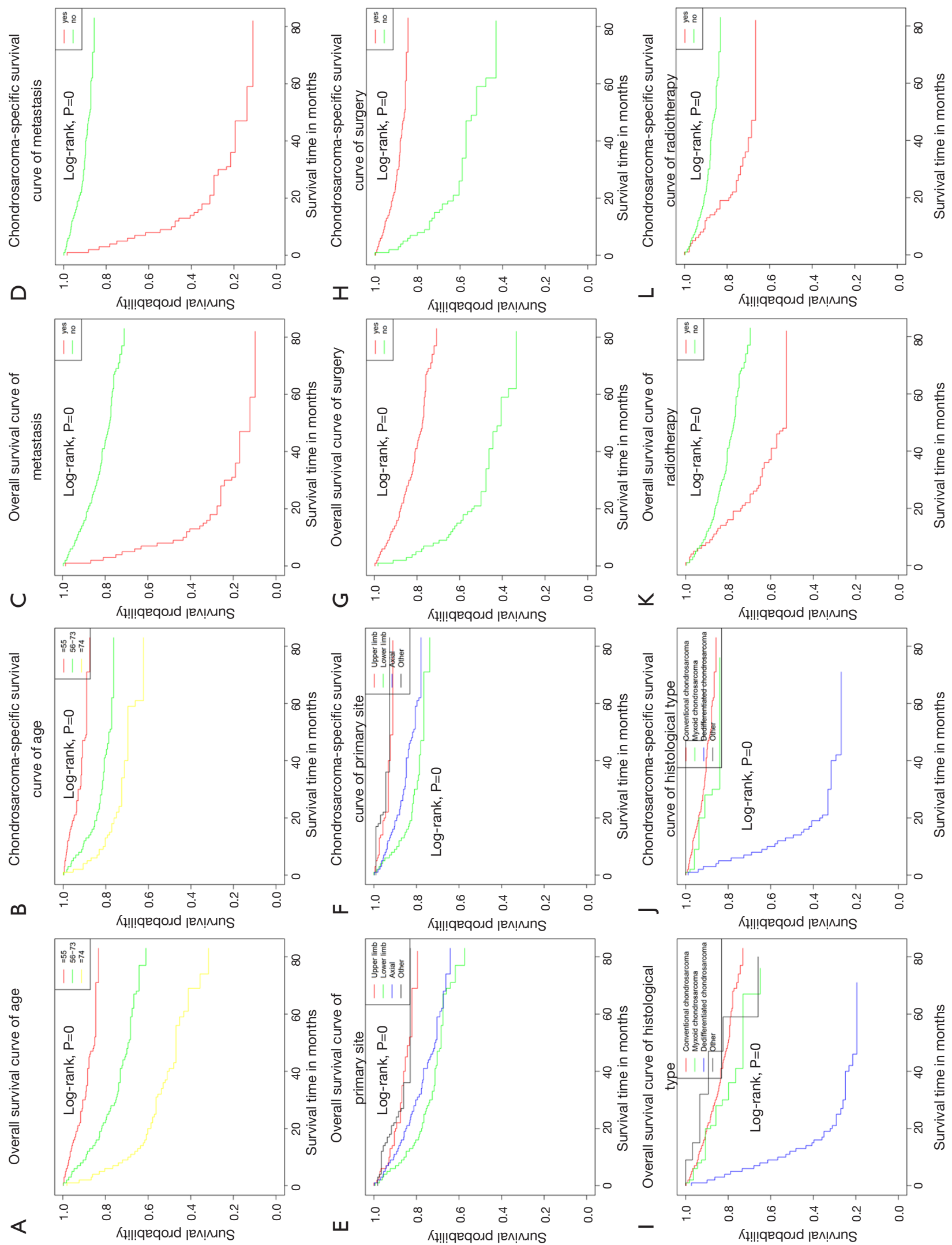

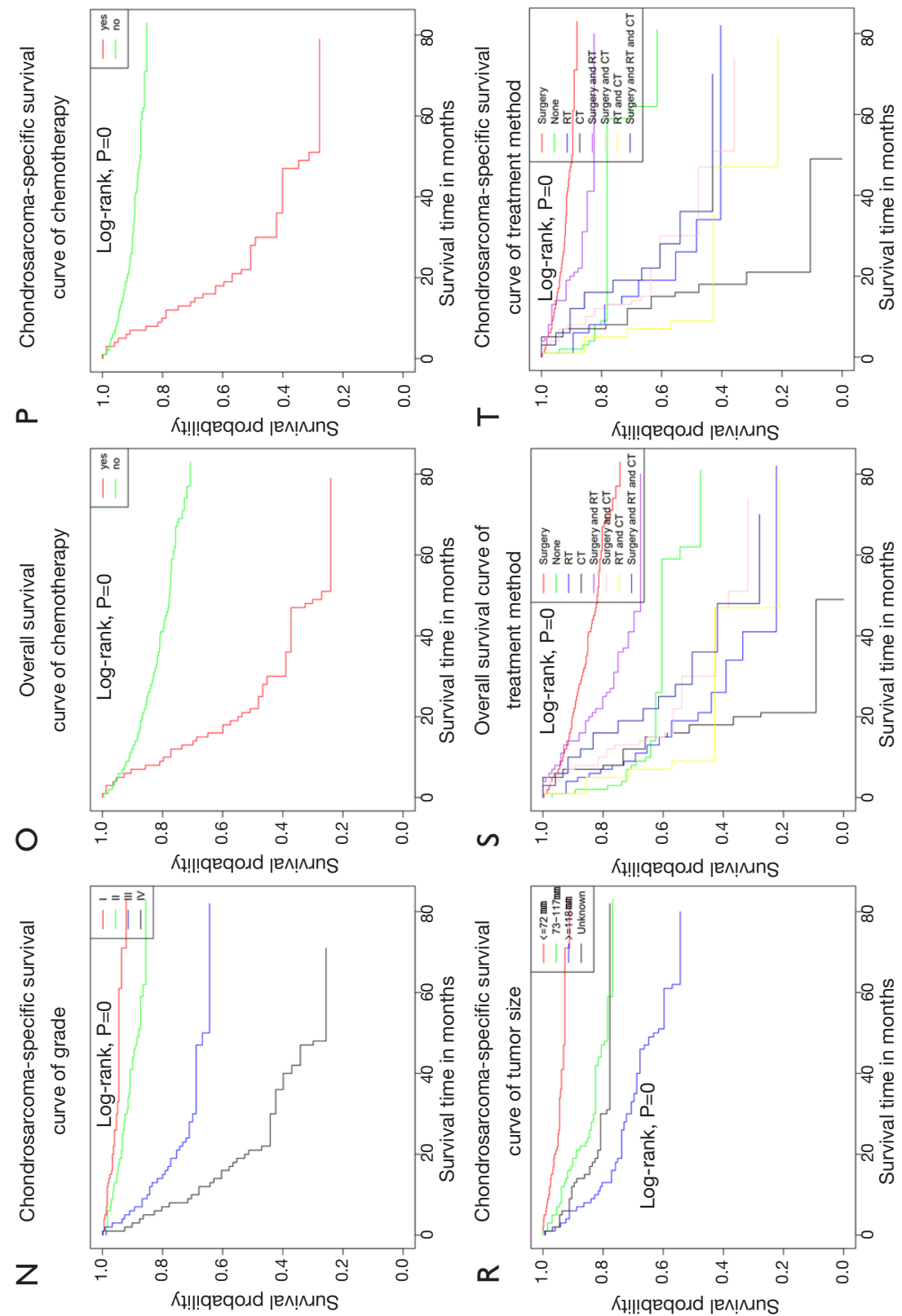

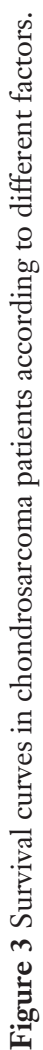


due to the different samples included, the differences in statistical methods, and the development of diagnostic and therapeutic techniques have led to somewhat different from the conclusions of previous studies. In the future, more samples and more detailed data will be needed to study the treatment of chondrosarcoma and to analyze the important factors affecting prognosis through big data analysis.

\section{Acknowledgments}

KC Hua: I would like to express my special thanks to my partners for the encouragement and support they gave me during my study. Thanks to my wife, Mrs. Sun, for her support for my life and research.

Funding: None.

\section{Footnote}

Reporting Checklist: The authors have completed the STROBE reporting checklist. Available at http://dx.doi. org/10.21037/tcr-20-357

Conflicts of Interest: Both authors have completed the ICMJE uniform disclosure form (available at http://dx.doi. org/10.21037/tcr-20-357). The authors have no conflicts of interest to declare.

Ethical Statement: The authors are accountable for all aspects of the work in ensuring that questions related to the accuracy or integrity of any part of the work are appropriately investigated and resolved.

Open Access Statement: This is an Open Access article distributed in accordance with the Creative Commons Attribution-NonCommercial-NoDerivs 4.0 International License (CC BY-NC-ND 4.0), which permits the noncommercial replication and distribution of the article with the strict proviso that no changes or edits are made and the original work is properly cited (including links to both the formal publication through the relevant DOI and the license). See: https://creativecommons.org/licenses/by-nc-nd/4.0/.

\section{References}

1. Rizzo M, Ghert MA, Harrelson JM, et al. Chondrosarcoma of bone: analysis of 108 cases and evaluation for predictors of outcome. Clin Orthop Relat Res 2001;(391):224-33.

2. Bus MPA, Campanacci DA, Albergo JI, et al. Conventional
Primary Central Chondrosarcoma of the Pelvis: Prognostic Factors and Outcome of Surgical Treatment in 162 Patients. J Bone Joint Surg Am 2018;100:316-25.

3. Reith JD, Horodyski MB, Scarborough MT. Grade 2 chondrosarcoma: stage I or stage II tumor? Clin Orthop Relat Res 2003;415:45-51.

4. de Camargo OP, Baptista AM, Atanásio MJ, et al. Chondrosarcoma of Bone: Lessons From 46 Operated Cases in a Single Institution. Clin Orthop Relat Res 2010;468:2969-75.

5. Björnsson J, Mcleod RA, Unni KK, et al. Primary chondrosarcoma of long bones and limb girdles. Cancer 1998;83:2105-19.

6. Murphey MD, Walker EA, Wilson AJ, et al. From the archives of the AFIP: imaging of primary chondrosarcoma: radiologic-pathologic correlation. Radiographics 2003;23:1245-78.

7. Guo W, Li D, Tang X, et al. Surgical treatment of pelvic chondrosarcoma involving periacetabulum. J Surg Oncol 2010;101:160-5.

8. Lee FY, Mankin HJ, Fondren G, et al. Chondrosarcoma of Bone: An Assessment of Outcome. J Bone Joint Surg Am 1999;81:326-38.

9. Giuffrida AY, Burgueno JE, Koniaris LG, et al. Chondrosarcoma in the United States (1973 to 2003): An Analysis of 2890 Cases from the SEER Database. J Bone Joint Surg Am 2009;91:1063-72.

10. Gelderblom H, Hogendoorn PCW, Dijkstra SD, et al. The Clinical Approach Towards Chondrosarcoma. Oncologist 2008;13:320-9.

11. Riedel RF, Larrier N, Dodd L, et al. The Clinical Management of Chondrosarcoma. Curr Treat Options Oncol 2009;10:94-106.

12. Bergh P, Gunterberg B, Meis-Kindblom JM, et al. Prognostic factors and outcome of pelvic, sacral, and spinal chondrosarcomas: a center-based study of 69 cases. Cancer 2001;91:1201-12.

13. Sheth DS, Yasko AW, Johnson ME, et al. Chondrosarcoma of the pelvis: Prognostic factors for 67 patients treated with definitive surgery. Cancer 1996;78:745-50.

14. Pring ME, Weber KL, Unni KK, et al. Chondrosarcoma of the pelvis. A review of sixty-four cases. J Bone Joint Surg Am 2001;83:1630-42.

15. Veth R, Schreuder B, van Beem H, et al. Cryosurgery in aggressive, benign, and low-grade malignant bone tumours. Lancet Oncol 2005;6:25-34.

16. Funovics PT, Panotopoulos J, Sabeti-Aschraf M, et al. Low-grade chondrosarcoma of bone: experiences from the 
Vienna Bone and Soft Tissue Tumour Registry. Int Orthop 2011;35:1049-56.

17. Chow WA. Update on chondrosarcomas. Curr Opin Oncol 2007;19:371-6.

18. Rhomberg W, Eiter H, Böhler F, et al. Combined radiotherapy and razoxane in the treatment of chondrosarcomas and chordomas. Anticancer Res 2006;26:2407-11.

19. Kalinski T, Krueger S, Sel S, et al. Differential expression of VEGF-A and angiopoietins in cartilage tumors and regulation by interleukin-1beta. Cancer 2006;106:2028-38.

20. Tan ML, Choong PFM, Dass CR. Anti-chondrosarcoma effects of PEDF mediated via molecules important to apoptosis, cell cycling, adhesion and invasion. Biochem Biophys Res Commun 2010;398:613-8.

21. Huang CL, Liu D, Nalano J, et al. Wnt-5a Expression Is Associated With the Tumor Proliferation and the Stromal Vascular Endothelial Growth Factor--An Expression in Non-Small-Cell Lung Cancer. J Clin Oncol 2005;23:8765-73.

Cite this article as: Hua $\mathrm{KC}, \mathrm{Hu} \mathrm{YC}$. Treatment method and prognostic factors of chondrosarcoma: based on Surveillance, Epidemiology, and End Results (SEER) database. Transl Cancer Res 2020;9(7):4250-4266. doi: 10.21037/tcr-20-357
22. Cooper GS, Virnig B, Klabunde CN, et al. Use of SEERMedicare data for measuring cancer surgery. Med Care 2002;40:IV-43-8.

23. Warren JL, Harlan LC, Fahey A, et al. Utility of the SEER-Medicare data to identify chemotherapy use. Med Care 2002;40:IV-55-61.

24. Bruns J, Elbracht M, Niggemeyer O. Chondrosarcoma of bone: An oncological and functional follow-up study. Ann Oncol 2001;12:859-64.

25. Fiorenza F, Abudu A, Grimer RJ, et al. Risk factors for survival and local control in chondrosarcoma of bone. J Bone Joint Surg Br 2002;84:93-9.

26. Bourgouin PM, Tampieri D, Robitaille Y, et al. LowGrade Myxoid Chondrosarcoma of the Base of the Skull. J Comput Assist Tomogr 1992;16:268-73.

27. Strotman PK, Reif TJ, Kliethermes SA, et al. Dedifferentiated chondrosarcoma: A survival analysis of 159 cases from the SEER database (2001-2011). J Surg Oncol 2017;116:252-7. 